\section{MK-2Q SECRETOS A VOCES DEL SOCIAL-MEDIA}
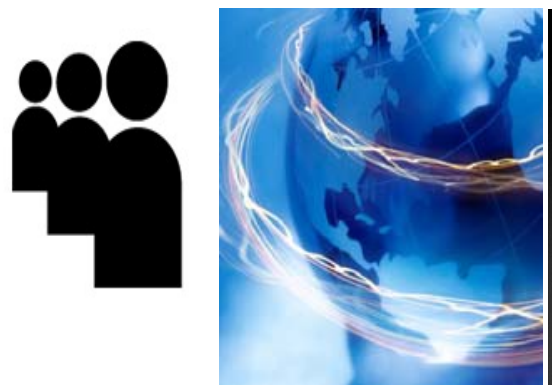

Pecvnia, Monográfico (2010), pp. 191-214

$M^{a}$ Aránzazu Sulé Alonso ${ }^{1}$ a.sule@unileon.es Universidad de León

Javier Prieto García javi.prieto.garcia@gmail.com

\title{
Resumen
}

Las redes sociales son un fenómeno emergente al que cada día se suman nuevos adeptos convirtiéndose en un sector exitoso que los expertos en marketing digital tienen que aprovechar. Esta investigación evidencia que la multidisciplinariedad entre ambas puede tener efectos muy beneficiosos y que su puesta en práctica es ya una realidad.

Pepsi, Starbucks, Avon, Adidas, infinidad de marcas de reconocido prestigio internacional han apostado muy fuerte por estos nuevos canales de actuación obteniendo resultados lo suficientemente reveladores como para hablar de nuevas modalidades de negocio, y aún más en este momento cuando parece que las formas de hacer marketing y publicidad convencionales están perdiendo protagonismo. Cada vez pasamos menos tiempo frente al televisor y más conectados a la red. Si una marca quiere encontrarnos, es probable que allí pueda hacerlo. Las redes sociales aportan grandes dosis de viralidad a los procesos de comunicación, las cuales son de gran calidad ya que, ¿hay mejores prescriptores que nuestros familiares y amigos?

Y todo esto para satisfacer a un agente que tiene mucho que decir: tu.

Palabras c lave: Redes sociales, marketing 2.0, facebook.

\section{Abstract}

Social media is an emerging phenomenon which wins new followers every day becoming a succesful sector that digital marketing experts have to make the most of. This research shows that multidisciplinarity between can have beneficial effects and its practice it's already a reality.

Pepsi, Starbucks, Avon, Adidas, and most of the well-known international brands have bet strongly for these new marketing chanels getting such good results that we can talk about new business methods and more evennow this moment where traditional marketing and

1 Facultad de Ciencias Económicas y Empresariales, Campus de Vegazana, s/n, 24071-León (España). 
advertising are losing prominence. Every day we spend less time in front of TV but more time surfing the internet. If a brand wants to find us it will probably find us there.

Social media contributes to get virality to the communication processes and of course quality because, are there better prescriptors than our relatives and friends?

And everythig to satisfy an agent that has a lot of to say: you.

Keywords Social media, marketing 2.0, facebook.

\section{1. ¿Qué es una red social?}

El hombre es un ser social. Siempre ha vivido en sociedad rodeado de sus semejantes, desde el origen de la especie hasta el momento actual. Y siempre ha mantenido relaciones de todo tipo con sus congéneres. Pero el paso del tiempo ha facilitado esta manera de interrelacionarse y comunicarse. Igual que el propio ser humano y las condiciones de vida del mismo han cambiado, las relaciones, y sobre todo, la forma de llevarlas a cabo,

192 también han sufrido alteraciones.

Esa interacción entre individuos ha dado un salto considerable desde el nacimiento de Internet (finales de los años 60), pero ese salto es todavía mayor a medida que termina el siglo $X X$ y comienza el siglo $X X I$. El nacimiento del PC (ordenador personal) y la proliferación de Internet (según un estudio de la consultora Gartner, a finales de 2009 la cifra estimada de hogares conectados era de 422 millones), como no podría ser de otra manera, han facilitado en gran medida el camino.

El hombre actual, del cual estamos cansados de escuchar que vive en un ambiente de estrés continuo en el que la falta de tiempo es una constante día tras día, cada vez recurre más a este medio ya no solo por la practicidad y versatilidad del mismo, sino porque le permite la posibilidad de realizar tareas más rápido, optimizando así uno de sus bienes más preciados, el tiempo. La posibilidad de hacer la compra a cualquier hora del día y de la noche, comprar entradas para el cine o para el teatro, incluso elaborar trámites administrativos, son solo algunos ejemplos del abanico de posibilidades que Internet pone a nuestra disposición. Por lo tanto, no es raro ni incomprensible el pensar que, si podemos mantener una "relación" con nuestro banco o con nuestro supermercado a través de la web, el relacionarnos con nuestros conocidos, familiares... sea infinitamente más sencillo. Bienvenido a la web 2.0.

\section{Bienvenido a la web $\mathbf{2 . 0}$}

Muchos son los que hablan de la web 2.0, pero antes de abordar este término, deberíamos delimitar a su antecesora, la web 1.0. Ésta, mucho menos mediática que la 2.0 por su evidente falta de atractivo frente a ésta, podríamos decir que define la forma de trabajar con las primeras webs: datos estáticos, sin interacción por parte del internauta, el cual acude a las web en busca de información. La relación termina en este momento, el proceso de comunicación es completamente unidireccional. Puede que hasta sea un concepto tan obsoleto que nos cueste trabajo comprenderlo, pero hasta hace relativamente poco la realidad era esta.

La web 2.0, en cambio, determina la existencia de una segunda generación de webs, basada principalmente en el término de comunidad o sociedad, y es aquí donde ya entra en juego el término de red 
social al igual que otros como blogs o wikis. Por lo tanto, el término web 2.0 engloba a toda aquella forma de relacionarse vía Internet que incluye interacción o intercambio de información entre las partes que conforman esa red, esa comunidad. Según la definición de Xabier Ribes, hablaríamos de 2.0 refiriéndonos a "todas aquellas utilidades y servicios de Internet que se sustentan en una base de datos, la cual puede ser modificada por los usuarios del servicio, ya sea en su contenido (añadiendo, cambiando o borrando información o asociando datos a la información existente), bien en la forma de presentarlos, o en contenido y forma simultáneamente". (Ribes, 2007)2. Antes de hablar de redes sociales enfrentémonos antes a los blogs. Un blog o bitácora no es más que un espacio en Internet en el que un individuo (bloguero o blogger) expone sus ideas, sus inquietudes, cualquier cosa a través de un texto o artículo también conocido como post, que se publica en la red y que por norma general se encuentra ordenado cronológicamente. El término proviene de la contracción de Weblog. Según publica Technorati.com en El Estado de la Blogosfera 2008 (State of the Blogosphere 2008), dicho año se cerró con 133 millones de blogs en todo el mundo que producían un millón de posts al día. Según este mismo informe, pero el relativo al año 2009 el perfil del bloguero es el que podemos observar en el Gráfico 1.

Como muestra el gráfico, la mayor parte de los blogueros son hombres entre 35 y 44 años, seguidos del rango de edad anterior, entre 25 y 34 años, pero podríamos afirmar que se trata de aproximaciones. Veamos un par de casos casi anecdó-

\footnotetext{
2 Disponible en:

http://sociedadinformacion.fundacion.telefo nia.com/telos/articuloperspectiva.asp@idarti culo $=2 \&$ rev $=73 . \mathrm{htm}$
}

ticos. El primero de ellos es el de Tavi Gevinson, una niña estadounidense que con tan solo 13 años es una de las blogueras más influyentes del mundo de la moda, tanto es así, que suele ocupar lugares destacados en los más importantes desfiles de moda del mundo. Comenzó con su blog Style Rookie (http://tavithenewgirlintown.blogspot.com hace apenas un año. Sus padres casi desconocían las aficiones de su hija hasta que ésta les pidió permiso para aparecer en el $\mathrm{New}$ York Times. Por otro lado, en mayo de 2009 falleció María Amelia López Soliño, probablemente la bloguera de mayor edad del mundo. Su blog (http://amis95. blogspot.com) fue un regalo de su nieto. A los 97 años de edad escribió su último post.

Estos son dos claros ejemplos de que la blogosfera (y la red en general) no entiende ni de edad, origen, raza, cultura o religión. El principal objetivo que todo bloguero persigue es dar su opinión, expresarse, compartir, y en muchos casos esperar una respuesta o feedback. En definitiva, comunicarse, interactuar. Hemos hablado del pasado con la web 1.0 y del presente con la web 2.0. Nuestra investigación se centrará en el marketing 2.0, al analizar la forma de hacer marketing en la 2.0. El futuro nos depara lo que en su día conoceremos como la web 3.0, la Web Semántica, de la cual se dice será más fácil de usar y más amigable, ya que entenderá el lenguaje coloquial de las personas y el camino necesario hacia la inteligencia artificial y además de ser la antecesora de la web 4.0, también conocida como la Web Ubicua. En este caso, se tratará de un modelo de omnipresencia de aplicaciones que dejarán los ordenadores personales y se implantarán en la red. 


\section{Gráfico 1}

\section{Perfil de los blogueros}

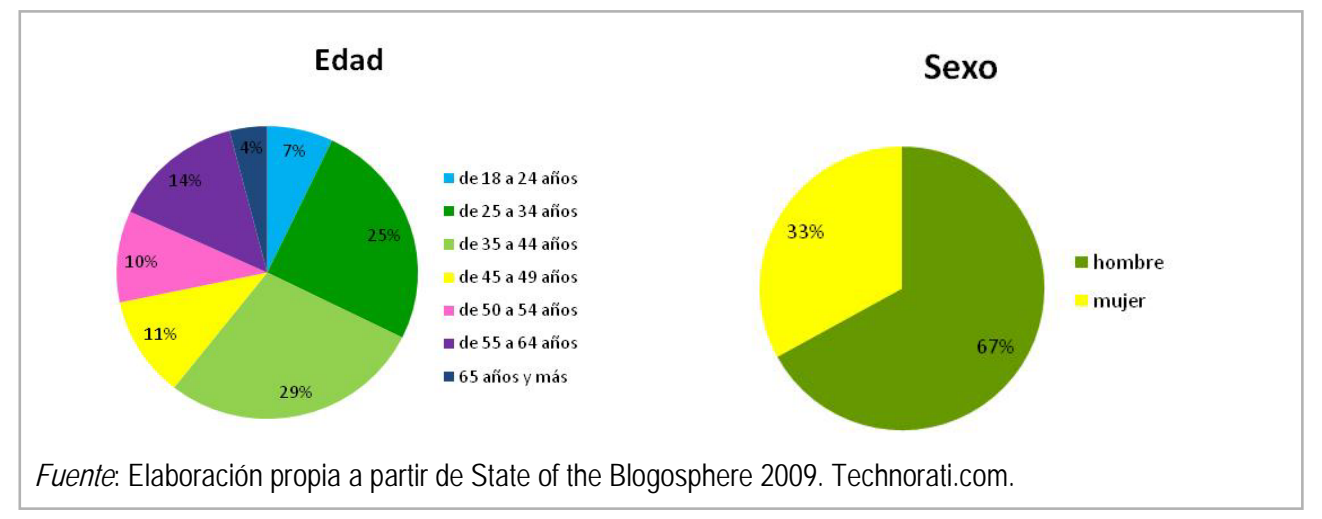

\section{Redes Soc iales: fundamentos y ońgenes}

Como hemos dicho, dentro de la web 2.0 se encuentran las redes sociales (Social Media o SRS-Sitios de Redes Sociales). Es un término muy extendido y popularizado que se encuentra en auge. Pero pongamos ejemplos, ¿a qué nos referimos al hablar de redes sociales? Muy probablemente muchos sepan ya a que nos referimos al plantear esta pregunta. Al hablar de redes sociales hablamos de Myspace, de Twitter, Tuenti, Facebook... todas ellas conocidas por muchos, pero no son las únicas (ni mucho menos), la lista es mucho más extensa. Sólo de habla hispana encontramos otras como por ejemplo Hi5, Networking Activo, Neurona, eConozco, Cielo, Qdamos, Spaniards, seguidos de un largo etcétera. Podemos decir sin miedo a equivocarnos que las redes sociales se están convirtiendo en un auténtico fenómeno que está revolucionando la forma de ver y de comportarse en Internet.

Pero al igual que para hablar del 2.0 abordamos primero el 1.0, antes de hablar de lo que hoy en día conocemos como una red social, explicaremos sus orígenes.
Se ha generalizado la idea de que el origen de las redes sociales se encuentra en la Teoría de Seis Grados de Separación (disponible en SixDegrees.com.), término acuñado en los años 90. Según esta teoría, todos los individuos del planeta estamos conectados a través de una cadena de tan solo cinco individuos o lo que es lo mismo seis separaciones, tal y como muestra el Gráfico 2, el individuo A y el individuo B se encuentran separados únicamente por cinco individuos, de manera que con tan solo seis saltos podrían ponerse en contacto. El fundamento de esta teoría es sustancialmente matemático, ya que el número de invididuos que conoce una persona puede ser más o menos fijo, pero a medida que avanzamos en esa cadena el número crece exponencialmente. Según esta teoría, cada persona cuenta en su círculo más cercano con aproximadamente cien personas (incluyendo familia, amigos, compañeros de trabajo...) y cada una de esas cien personas conoce a otras cien personas, por lo que con tan solo dos pasos ya nos pondríamos en contacto con diez mil. Cada uno de esos diez mil individuos conoce a otros cien más, ampliando el círculo a un millón. En un cuarto salto llegariamos a cien millones, diez mil millones en un quinto nivel y un millón de millones en el sexto y último 
paso. Es evidente que cuanto más pasos haya que dar, más dificil será la comunicación entre los individuos, pero Internet pone a disposición del usuario infinidad de herramientas para que este proceso sea más sencillo. Por lo tanto ya podemos vislumbrar una primera aproximación a lo que conocemos como redes sociales. ¿Por qué Redes Sociales?, y, ¿por qué ahora?

\section{Gráfico 2}

\section{Seis grados de separación (Six Degrees)}

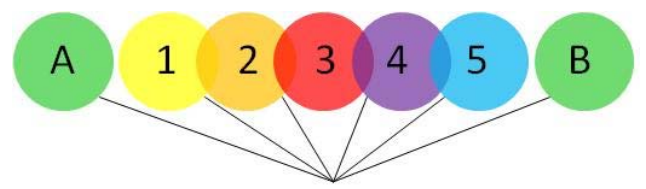

Seis Grados de Separación

Fuente: Elaboración propia a partir del original de Laurens van Lieshout a través de la Licencia de documentación libre de GNU disponible en http://es.wikipedia.org/wiki/Archivo:Six_degrees_of_separation.png

\section{Imagen 1}

\section{Evolución de los medios}

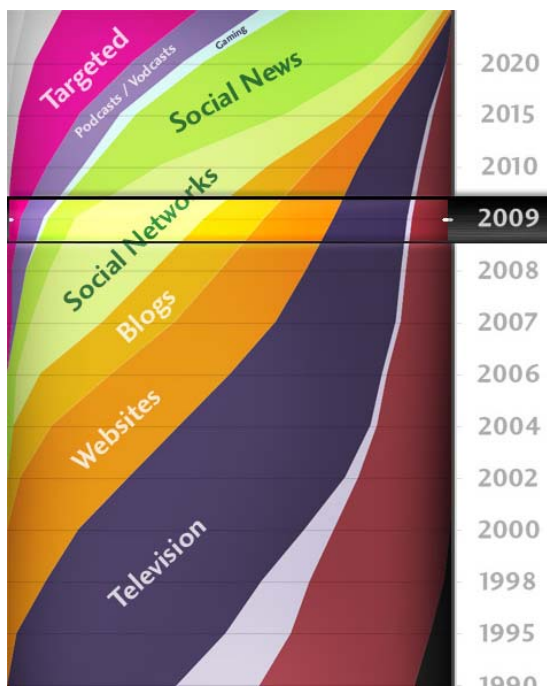

Fuente: www.fernandoplaza.com/2009/06/las-redessociales-son-los-centros-comerciales-de-intenet.asp
Estamos en el momento justo, el año 2009 se ganó con creces el título de año de los bienes virtuales. Según el Informe de la Sociedad de la Información en España 2009 elaborado por Telefónica, el uso durante este último año de las redes sociales se ha incrementado en un $430 \%$, situando a España en el segundo país por detrás de Reino Unido, según la tasa de penetración, que en el caso español es de aproximadamente el $75 \%$, y es que en España hay aproximadamente 18 millones de perfiles creados en las diferentes redes sociales.

Si hablamos en términos generales, España ocupa el séptimo lugar según el número de usuarios (casi 20 millones) pero el quinto según el número de horas inmerso en los social media tal y como muestra la siguiente Tabla 1.

De estos 18 millones de usuarios, los jóvenes entre 16 y 24 años, son los que más cuentas han abierto durante este último año, con un incremento del $500 \%$, con respecto al año anterior. Para 
este segmento de población es tan importante el uso de estas herramientas que ya son consideradas como una prolongación de la vida adolescente. Esto demuestra que las redes sociales se han introducido en el momento justo, cuando el desarrollo tanto social como tecnológico era el adecuado.

\section{Tabla 1}

\section{Uso de los Social Media}

\section{País}

\section{$1^{\circ} \quad$ Estados Unidos}

$2^{\circ} \quad$ Japón

$3^{\circ} \quad$ Brasil

$4^{\circ} \quad$ Reino Unido

$5^{\circ} \quad$ Alemania

$6^{\circ} \quad$ Francia

$7^{\circ} \quad$ España

8 Italia

$9^{\circ} \quad$ Australia

$10^{\circ} \quad$ Suiza
Audiencia (miles)

142.052

46.558

31.345

29.129

28.057

26.786

19.456

18.256

9.895

2.451
Tiempo por persona

6:09:13
$2: 50: 21$
$4: 33: 10$
$6: 07: 54$
$4: 11: 45$
$4: 04: 39$
5:30:55
$6: 00: 07$
$6: 52: 28$
$3: 54: 34$

Fuente: Elaboración propia a partir de www.gabycastellanos.com/espana-el-septimo-pais-en-uso-de-socialmedia/ - The Nielsen Company

Las redes sociales ya forman parte de nuestras vidas, pero no sólo de las nuestras (seres humanos), sino también de la vida de las marcas.

Veamos un caso cuanto menos sorprendente. La multinacional Pepsi, ha mostrado su convencimiento acerca de la efectividad de la publicidad y su repercusión en que alguien tome un refresco de cola o de otro sabor. Así lo constató Indra K. Nooyi, en la siguiente declaración: "La gente no tomara mas Pepsi porque pongamos mas publicidad convencional (vallas) en la calle. El sabor es subjetivo, puede gustar o no. No vamos a cambiar la mente de nadie diciéndoles que tomen Pepsi. La gente debe tomar sus propias decisiones". Pero, sin embargo, recientemente se ha sabido que ha destinado 20 millones de dólares a un proyecto dentro del Social Media bajo el nombre The Pepsi Refresh Project. ¿Locura? ¿Tendencia?

\subsection{Perfil de usuario}

Según la agencia inglesa Ofcom, podemos dividir en dos grupos al grueso de la población, según sean o no, usuarios de redes sociales. Empezando por los no usuarios de redes sociales distinguimos tres grupos:

- Preocupados por la seguridad: desconfían de quién pueda acceder a su perfil, que su información personal sea revelada y del uso que de ésta se pueda hacer.

- Inexperiencia técnica: no familiarizadas con el uso de ordenadores y de Internet.

- Rechazo intelectual: no les interesan las redes sociales y consideran que su uso supone una pérdida de tiempo.

Si por otro lado, consideramos a aquellos que sí hacen uso de estas redes, el estudio los clasifica en cinco grupos: 
- Socializadores alfa: utilizan las redes sociales para conocer a otra gente y divertirse. Son un grupo minoritario.

- Buscadores de atención: crean su perfil, publican fotos... en busca de atención y comentarios de los demás. Suponen un grupo más amplio que el anterior.

- Seguidores: formado por usuarios que se conectan para seguir la actividad de sus contactos. Es uno de los grupos mayoritarios.

- Fieles: de los grupos más populares junto con el anterior, incluye personas que usan las redes sociales para encontrar viejos amigos.

- Funcionales: utilizan las redes sociales ocasionalmente para propósitos más concretos y particulares.

Existen otras clasificaciones de los usuarios de SRS, como por ejemplo la elaborada por Forrester Research Inc., que divide a los usuarios en seis grupos: creadores (creators: es el grupo más participativo de todos, sus integrantes publican páginas web, mantienen blogs y utilizan youtube), críticos (critics: de gran importancia para las marcas, comentan y dan su opinión sobre productos y servicios, tienen mucho impacto en el público, desde un punto de vista comercial, contar con su apoyo puede ser muy rentable), coleccionistas (collectors: usuarios muy activos que conocen muy bien el funcionamiento de la red), participadores (joiners: su principal propósito es utilizar las redes con fines únicamente sociales), espectadores (spectator: usuarios activos pero que intentan mantenerse al margen) y por último, los usuarios inactivos (inactives: que no realizan ninguna de las actividades anteriores).

Los porcentajes representativos de cada segmento considerado se muestran en el Gráfico 3, para el caso de España y referidos a datos de 2009 .

\section{Gráfico 3}

Clasificación (\%) de usuarios según clasificación de Forrester Research Inc.

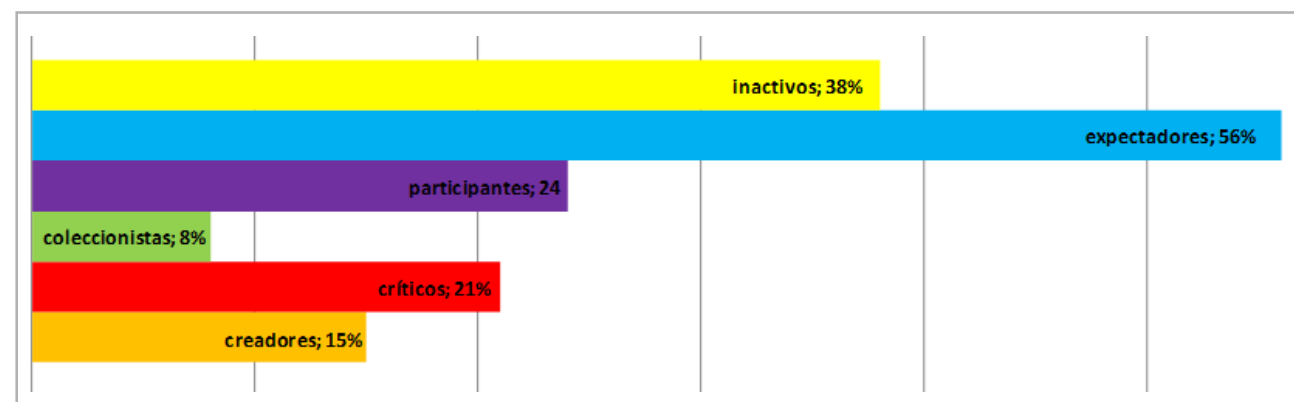

Fuente: Elaboración propia a partir de la aplicación de Forrester Research Inc. disponible en www.forrester.com

\subsection{Hablemos de redes sociales}

A continuación, se explican las redes sociales que cuentan con mayor aceptación en España.

\section{(i) tuenti}

3.2.1. Tuenti: la red de los jóvenes

Tuenti (tuenti.com) puede que sea la red social de los jóvenes por excelencia en España. Nació en el año 
2006 de la mano de su creador, Zaryn Dentzel; un estadounidense afincado en Madrid que en enero de ese año decidió crear una red en principio dirigida a estudiantes, pero debido a su más que notable éxito, decidió abrirla al público en general. Tuenti es una red social de acceso restringido a la que solo se puede acceder mediante una invitación que cualquier usuario activo de Tuenti puede enviar a cualquiera de sus contactos.

Cada usuario cuenta con una página denominada "Mi perfil" en la que puede compartir toda aquella información que desee sobre él mismo, por ejemplo nombre, edad, fotografía, fecha y lugar de nacimiento, estado civil, sitios web, estudios, intereses... Tuenti ofrece la posibilidad de subir fotos en las que se puede etiquetar a los individuos que aparecen en ellas. Además estas fotos pueden ser comentadas. En el año 2009, el chat llegó a Tuenti, ofreciendo así un servicio adicional que permitía conversaciones en tiempo real entre los usuarios conectados a la red.

Al margen de lo que se pueda pensar, Tuenti no proviene del inglés twenty (veinte) sino que fue el resultado de la búsqueda de un nombre que incluyera las palabras "tu" y "ti". Finalmente se eligió Tuenti porque sonaba bien, incluía ambas palabras y además era una abreviatura de "tu entidad".

El número de usuarios ${ }^{3}$ de Tuenti es uno de los secretos de la compañía. Según aseguran desde la red española, en Tuenti se apuesta más por la calidad que por la cantidad, pero aún así algunos hablan de

${ }^{3}$ Disponible en:

http://www.elpais.com/articulo/portada/red /social/espanola/compite/Facebook/estudia ntes/elpeputec/20080410elpcibpor_1/Tes; http://blog.mosen.es/2009/07/estadisticassobre-la-red-social-tuenti.html.
5 o 6 millones de usuarios, otros de menos, tan solo 4 millones, pero incluso hay otros que aseguran que supera a Facebook en número de usuarios. Si bien, debemos tener en cuenta las limitaciones geográficas de la red española.

Como ejemplos de la utilización de Tuenti, podemos destacar la masiva reacción de los usuarios de la red ante el secuestro de Marta del Castillo, con la creación de grupos de apoyo que mostraban su solidaridad con la familia. El actual Lehendakari Patxi López también hizo uso de esta red para acceder al voto de los jóvenes. Además de estos casos especiales, marcas de gran consumo, de moda y grupos de música entre otros, también utilizan esta plataforma como escaparate, ya sea mediante la creación de eventos o directamente con anuncios que aparecen mientras el perfil del usuario se está cargando.

Prueba de ello, ha sido la campaña navideña que pretendía atraer a los usuarios de Tuenti regalándoles "tuentilacasitos". Un usuario afirmaba que le llamaba la atención que le enviaran a su casa "tuentilacasitos", es decir, Lacasitos, que en vez de tener impreso en el envase la etiqueta de Lacasitos, tenía el nombre de la red social Tuenti. Otro hecho que ha pretendido aumentar la notoriedad de esta red social tuvo lugar en el concierto del cantante venezolano Carlos Baute, en El Patio del Conde Duque (Madrid), dónde dicho cantante, entre canción y canción, se puso una camiseta en la que ponía Tuenti. Su repercusión tuvo como consecuencia que ese mismo día se crearan muchos perfiles nuevos.

\subsubsection{MySpace: espacio personal}

\section{Q⿱⺈ Myspace}

(myspace.com) es similar al de Tuenti aunque de mayor envergadura. Myspace nace en 2003 y desde entonces, su 
popularidad ha crecido enormemente. La oferta de servicios que MySpace pone al alcance de los usuarios es muy extensa.

Aparte de mandar mensajes y subir fotos, MySpace también permite el acceso a foros de debate, así como crear blogs y editar las páginas, en definitiva, permite una completa personalización del espacio, de ahí su nombre. Myspace cuenta con multitud de plataformas y aplicaciones como la creación de boletines y grupos, un servicio de mensajería instantánea (chat) o un apartado de vídeos, entre muchos otros. Los perfiles contienen varias secciones que muestran los datos de cada usuario, pero MySpace ofrece también algo diferente. Muchos grupos de música y artistas independientes noveles utilizan esta red para dar a conocer sus trabajos. Myspace permite a los artistas la posibilidad de subir seis pistas de audio en formato $\mathrm{mp3}$.

Al igual que el resto de redes, MySpace también utiliza la publicidad, tanto de perfiles creados en su seno como de enlaces exteriores. MySpace supone una auténtica revolución social, sobre todo en Estados Unidos ocupando uno de los lugares más visitados de la red.

\subsubsection{Twitter: en pocas palabras}

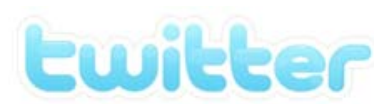

Twitter

(twitter.com)

es una de las redes sociales

más populares en todo el mundo junto con Facebook. Principalmente podemos decir que Twitter es una red basada en un servicio de micro-blogging, esto significa que los usuarios de Twitter pueden escribir pequeñas entradas o reseñas (denominadas tweets) de 140 caracteres como máximo que comparten con sus amigos o con el resto del mundo si así lo desean.
Twitter nació en 2006 en Estados Unidos y al igual que sus compañeras, lo que en un principio surgió como una red interna, finalmente se abrió al público en general y se extendió por gran parte del mundo. Pero si algo caracteríza a Twitter al margen de sus famosísimos tweets es la tecnología que utiliza esta red social, vinculando la web con teléfonos móviles a través de servicios de mensajería sms, programas de mensajería instantánea como MSN o incluso mediante Facebook. Actualmente Twitter representa uno de los sistemas de comunicación más utilizados en la red, y no sólo como medio de comunicación entre familiares y amigos, sino también como medio para establecer contactos profesionales.

Se estima que el número de usuarios de Twitter experimente un crecimiento sostenido que incremente hasta 15 veces su tamaño anualmente. Pero a pesar de que este crecimiento es considerable (es la red social que crece más rápidamente), según RJMetrics, del total de usuarios de Twitter, que ronda los 75 millones de cuentas, tan sólo un $17 \%$ escribieron algún tweet en diciembre de 2009, el $25 \%$ no tiene seguidores (usuarios que siguen las modificaciones de una cuenta), y el $40 \%$ no ha escrito ni un solo tweet. Esto pone de manifiesto que de esas 75 millones de cuentas, 30 millones nunca han sido actualizadas, por ello, para frenar esta tendencia, se están desarrollando nuevas funciones y aplicaciones para dotar de un mayor atractivo a esta red social. El valor actual estimado de Twitter se sitúa en mil millones de euros.

El número de usuarios registrados de Twitter también es todo un misterio ${ }^{4}$,

4 Disponible en: http://www.marketingdirecto.com/noticias/37685-ocho-cada-diezusuarios-utilizan-twitter-para-establecercontactos-profesionales-. 
aunque recientemente según un estudio de la consultora Sysomos, se desvelan los porcentajes aproximados de participación a partir de una muestra de 16 millones de usuarios. Así los tres primeros países con respecto al uso de Twitter son Estados Unidos, Brasil y Reino Unido con un $50.88 \%$, un $8.79 \%$ y un $7.20 \%$ respectivamente. España ocupa el décimo cuarto lugar después de otros países como Filipinas, Japón o India ${ }^{5}$. Aunque sí que podemos decir que el $86 \%$ de los usuarios de Twitter, según un estudio realizado por Madrid Network, esta red social es vista como un espacio que favorece la colaboración entre profesionales, donde se favorece la creatividad y las ideas y propuestas fluyen.

Con respecto a su relación con las marcas, en un principio Twitter prescindía de ellas, aunque esta relación era desigual en sentido contrario. Muchas marcas acudían a esta red social (al igual que a otras) guiadas por el impulso de aumentar su valor añadido y mejorar su imagen al prestar un servicio de comunicación en tiempo real. Pero en septiembre de 2009, se produjo un giro cuando desde Twitter se modificaron los términos de uso y anunciaron la inclusión de publicidad en sus servicios, que previsiblemente comenzará a utilizarse en 2010, eso sí, Stone, uno de los fundadores de la red, asegura que será una publicidad totalmente diferente a todas las formas de hacer publicidad online tradicionales.

\section{E caso de Facebook

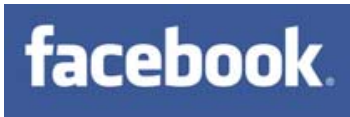

http://interactivaweb.com/2010/01/16/me xico-espana-y-chile-con-mas-presencia-entwitter/

${ }^{5}$ Disponible en:

http://interactivaweb.com/2010/01/16/me xico-espana-y-chile-con-mas-presencia-entwitter/
El caso de Facebook (facebook.com) es uno de los más increíbles y apasionantes en lo que a redes sociales se refiere. Mark Zuckerberg, estudiante de la Universidad de Harvard, fue su creador en el año 2004, motivo por el cúal, inicialmente estaba destinada en exclusiva a los estudiantes de dicha universidad pero, desde 2006 está disponible para todo aquel usuario que posea una cuenta de correo electrónico.

Originalmente, los facebooks son publicaciones que hacen las universidades americanas al comenzar el curso. Estos contienen las fotografías y los nombres de los estudiantes, siendo su principal objetivo el ayudar a que éstos se conozcan. Facebook no es más que esta idea trasladada a la web en el momento oportuno y que debido al apoyo necesario se ha extendido a todo el público.

¿Qué tiene Facebook que lo hace tan especial?. Algunos incluso se atreven a decir, que en la historia de la computación primero se creó el ordenador personal, luego Windows, a continuación Internet y después Facebook. Zuckerberg ha llegado a definirlo como el sistema operativo social de la web.

Según un Estudio sobre Redes Sociales en Internet elaborado por Elogia Ipsofacto para el Interactive Advertising Bureau (IAB Spain), en España más del $60 \%$ de los usuarios de redes sociales accede a su perfil a diario, y casi el $85 \%$ lo hace al menos una vez a la semana, es más, el estudio también revela que el tiempo que pasan conectados es superior al que pueden pasar, por ejemplo, leyendo un diario digital. Del total de encuestados Facebook es utilizada por el $82 \%$ de la muestra, seguida por Tuenti con un $24 \%$.

La razón es muy simple, interacción: envío de mensajes y fotografías a amigos que a su vez otros amigos pueden ver, comentar y responder, todo esto en tiempo real y de 
forma gratuita en la mayoría de los casos. Pero esto no es muy diferente a lo que ofrecen otras redes...

Puede que uno de los elementos culpables del éxito de Facebook entre la sociedad sea las conocidas aplicaciones. Facebook ofrece algo más que otras redes no hacen $y$, es precisamente esto: la posibilidad de hacer tests cuyos resultados puedas compartir con tus contactos o incluso, aplicaciones con las que jugar en red con ellos. La lista no tiene fin. Según tufacebook.com con fecha 19 de febrero de 2009, Facebook tiene 52.000 aplicaciones, y cada día se crean en torno a 140 nuevas. Con respecto al número de Usuarios Activos Mensuales (UAM), la aplicación más popular es Farmville, con más de 60 millones de UAM (juego en el que se cultivan frutas y verduras y se crían animales), seguido de Causes con 32 millones de UAM (en el que los usuarios crean o se afilian a causas que creen conciencia $\mathrm{o}$ provoquen un impacto positivo en el mundo, por ejemplo, ayuda a los damnificados por el terremoto de Haití).

Además, Facebook ofrece la posibilidad de que el usuario cree una página o grupos de los que el resto de usuarios pueden hacerse miembros, y puede ser (ipor qué no?) que aquí tambíen se encuentre el quiz de la cuestión. Ejemplos de esto son páginas como la de Michael Jackson en Facebook con más de diez millones y medio de fans en todo el mundo, Barack Obama con más de siete millones, CocaCola con más de cuatro millones, o la crema de chocolate Nutella con casi tres millones y medio de fans.

Pero, no siempre es tan fácil alcanzar popularidad en Facebook. Esto lo demuestra un estudio de la compañía de análisis especializada en redes sociales, Sysomos, que después de revisar 600.000 páginas de usuario de Facebook obtuvieron los resultados que se muestran en el Gráfico 4.

\section{Gráfico 4}

\section{Seguimiento de páginas en Facebook}

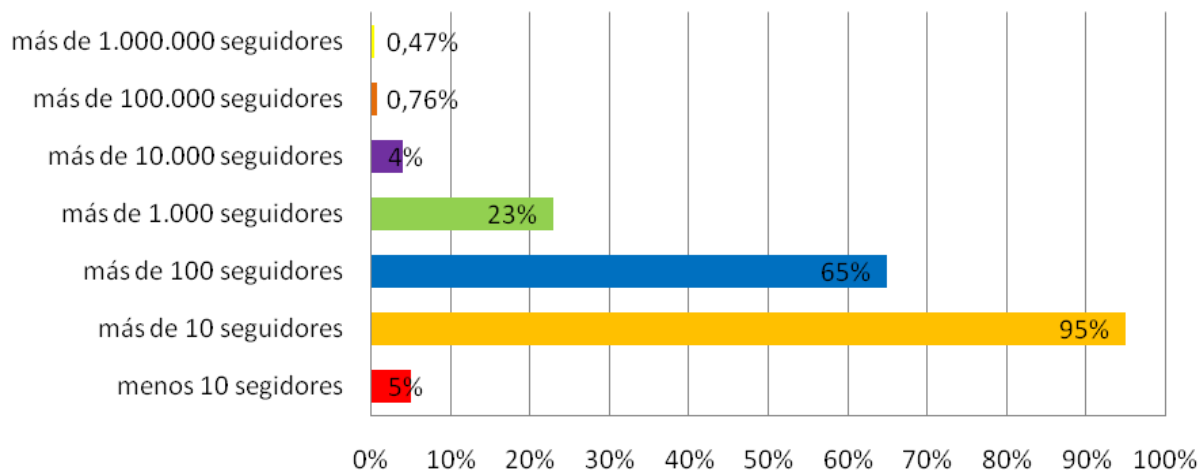

Fuente: Elaboración propia a partir de la información disponible en www.theinquirer.es/2009/11/29/no-es-facilser-popular-en-facebook.html-Sysomos

Esta interacción se está convirtiendo en un arma de doble filo para Facebook y para otras redes sociales. Esa apertura al mundo puede implicar una pérdida de intimidad, de ahí que el informe publicado por la Agencia Española de Protección de Datos (AEPD) y el Instituto Nacional de Tecnologías de la Comunicación (INTECO) 
advierta de los riesgos para mantener la privacidad y seguridad de los usuarios de las redes sociales.

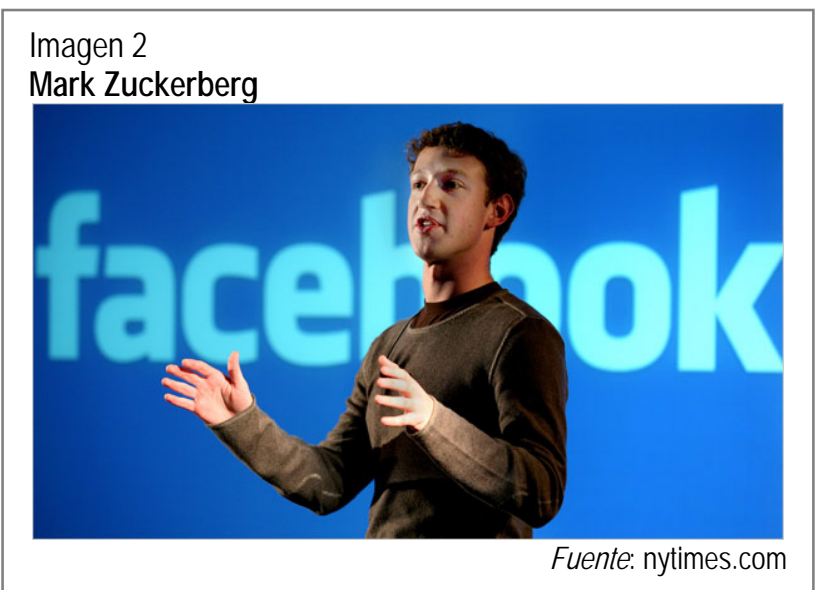

Como consecuencia, Mark Zuckerberg ha publicado una carta abierta dirigida a todos los usuarios de Facebook, disponible en el propio sitio de Facebook, dónde explica la evolución que ha experimentado esta red social. Como ya hemos dicho, en un principio Facebook estaba dirigida a estudiantes que, reunidos en comunidades, compartían material, fotografías, información más o menos privada que estaba disponible, podemos decir, únicamente para ellos. Al popularizarse, hoy más de 350 millones de personas
utilizan Facebook, el tamaño de esas comunidades ha crecido enormemente, por lo que esa privacidad empieza a cuestionarse, y es lógico si pensamos que redes que antes tenían un número más o menos limitado de usuarios ahora tienen millones.

Por lo tanto, desde Facebook se ha llegado a la conclusión de que quizá este formato no sea el más conveniente para garantizar esa ansiada privacidad.

Como respuesta han lanzado un modelo nuevo infinitamente más simple que el de las redes, dónde cada usuario puede indicar el nivel en el que el contenido que comparte en la web está disponible, sólo para amigos, amigos de amigos, todo el mundo... pudiendo de esta forma filtrar y controlar sus movimientos.

Como prueba de la confianza que Zuckerberg tiene en el nuevo sistema de privacidad de Facebook, su perfil en la red social se encuentra abierto a todo el mundo. Él mismo dice: "a los que preguntan, abrí la mayoría del contenido en mi página personal de Facebook para que las personas puedan verlo. Puse algunas cosas privadas, pero no vi la necesidad de ponerle un límite a fotos con mis amigos, familia u osito de peluche".

Según un estudio elaborado por Google Zeitgeist, entre los cuatro términos buscados a nivel global que más han crecido en Google durante el año 2009 se encuentran Facebook, Tuenti y Twitter en segundo, tercer y cuarto lugar respectivamente (el primer lugar lo ocupa Michael Jackson). En España entre los emergentes aparece Facebook en primer lugar, mientras que la lista de los más populares la encabeza Tuenti.

Después de este análisis, parece lógico pensar que tantos millones de personas de todo el mundo no pueden estar equivocadas, ¿no?. Pero además, debemos tener en cuenta que, cuando un soporte es respaldado por tal cantidad de individuos, el considerarlo en términos de rentabilidad publicitaria tampoco ha de resultarnos extraño, y más cuando un medio como es 
Internet ya supera en preferencias al móvil o a la televisión para niños y adolescentes, según el Foro Generaciones Interactivas, y en un momento en el que el $81 \%$ de los usuarios de la red recuerda alguna campaña de publicidad gráfica en Internet, según el Estudio de Eficacia de Formatos Publicitarios de Display, elaborado por The Cocktail Analisys para IAB Spain.

Es tal el uso de estos instrumentos, que en Indonesia, Facebook y Twitter, son utilizados para evitar los atascos. Mediante un logaritmo que toma los datos de más de 2000 taxis y de partes de la Policía, se dibuja un mapa donde las calles son de color verde, ámbar o rojo dependiendo del nivel de congestión vial. El resultado de este logaritmo puede consultarse a través de estas redes.

Se constata que su ámbito de aplicación aumenta progresivamente $y$ es multidisciplinar. El Departamento de Bomberos de Los Ángeles también utiliza Twitter para actualizar en tiempo real los partes de noticias relacionadas con accidentes, fuegos, catástrofes naturales... Y así mantener informada a toda la comunidad. Aunque no todos los usos de las SRS son políticamente correctos, ya que por ejemplo, Twitter se utiliza en Texas y en la Ciudad de México para que los conductores eviten los controles de alcoholemia.

Todos estos casos reales evidencian que las redes sociales son un fenómeno emergente al que se suman nuevos adeptos convirtiéndose en un sector exitoso que los expertos en marketing digital tienen que aprovechar.

\section{Fac ebook y las marcas}

El pasado mes de septiembre de 2009, Mark Zuckerberg hablaba en su blog sobre la rentabilidad de la red social que preside, Facebook. Exponía lo siguiente: "a comienzos de este año dijimos que esperábamos tener un flujo de caja positivo en algún momento de 2010. Me complace informar que hemos logrado ese hito".

Es evidente que tanto Facebook como cualquier otra red social une personas. Personas que en principio tienen una serie de intereses y cualidades en común o que simplemente se conocen. Personas a las que les gustan las mismas cosas. En definitiva, o dicho de otra manera, grupos de compradores potenciales que voluntariamente se agrupan e intercambian opiniones, y acaso ino son nuestros familiares, amigos y conocidos nuestros mejores prescriptores?.

¿Qué pasa cuando una marca se da cuenta de esto?. Ni que decir tiene que la mayor parte de marcas de gran consumo tienen su página en Facebook, cuyo creador (vinculado a la marca o no) la actualiza periódicamente y cuyos miembros hacen comentarios acerca de la marca o del producto, suben fotos y vídeos, en definitiva, establecen canales de comunicación de una importancia inmensurable para el branding. Valoraciones sobre nuevos productos, críticas, recomendaciones, todo vale. Aún así, las empresas deben actuar con extrema cautela en lo relativo a fines comerciales, puesto que la AEPD establece que:

- El sistema de Protección de Datos se basa en el consentimiento del usuario a la hora de recibir cualquier tipo de comunicación.

- Únicamente en dos supuestos será posible comunicarse con un individuo: en el caso de que sólo se comunique la existencia de la empresa sin fin comercial alguno, o cuando se mantenga el carácter comercial pero las comunicaciones vengan "elaboradas por un tercero y sin contraprestación económica".

Facebook es mucho más que una forma de contactar entre amigos y de compartir 
contenidos privados. Además de todo lo expuesto, también permite testar los últimos lanzamientos de los principales anunciantes, y todo ello gratis. Estamos aludiendo a Bloguzz, una de las aplicaciones más utilizadas. Gracias a ella, los usuarios reciben gratuitamente productos, muestras, invitaciones... a cambio de opiniones. Evidentemente, la compañía está detrás, no es una simple página creada por fanáticos de la marca. En la página de Bloguzz de Facebook podemos leer: iBienvenido a Bloguzz! La primera aplicación que permite a los usuarios de la red social probar productos y opinar sobre ellos. iDesde conducir un Aston Martin V8 Vantage hasta asistir en primicia a un concierto del grupo Pereza, pasando por la lectura del último libro de Isabel Allende o testear el nuevo maquillaje de Max Factor!. Entre los clientes de Bloguzz están Carrefour, Coca-Cola, Nike, Muji, Honda, Levi's, Max Factor o Gillette.

Bloguzz no es la única plataforma de apoyo a las marcas en Facebook, también existe Tiickr, la primera empresa española dedicada únicamente a los usuarios de Facebook. Para poder participar en esta nueva iniciativa se requiere ser miembro de Facebook así como de Twitter o Tuenti. Tiickr tiene como misión dar a probar los últimos lanzamientos de las marcas, a cambio de comprometerse a aportar opiniones objetivas, estar interesado en innovar, comunicar y probar productos, y todo para garantizar una promoción mediante el boca-oreja de una manera "efectiva, medible y económica", según señala Tiickr.

El primer anunciante de Tiickr ha sido Fon, la famosa comunidad de conexión Wifi gratuita creada por Martín Varsavsky en 2006. El experimento consistía en dar la posibilidad a 2.000 usuarios de opinar sobre los servicios de Fon de forma gratuita. De esos 2.000, 100 pasarían a una segunda fase, de los cuáles tan solo 6 pasarían a una tercera y última fase en la que recibirían varios premios y artículos de la tienda Fon.

\subsection{Casos reales}

Realmente, las redes sociales están revolucionando la forma de ver y hacer marketing en la red. Es más, podemos afirmar que es una de las nuevas herramientas a disposición de éste de mayor importancia y envergadura en la actualidad. Si lo que más nos interesa es llegar al cliente, la probabilidad de encontrarle en una red social es sustancialmente elevada.

Las marcas cada vez son más conscientes de todo este fenómeno y por eso cada vez invierten mayores cantidades de dinero en este nuevo medio. Aparte de los casos ya vistos anteriormente, muchas otras marcas de reconocido prestigio internacional han apostado por el social media. Algunos ejemplos son:

Avon, empresa de cosmética y productos de belleza para la mujer, y concretamente Mark, su marca dirigida al target juvenil, ha sabido darse cuenta de esto y a finales de 2009, ha inaugurado un espacio de comercio electrónico dentro de la red social (http://www.facebook.com/mark-girl) aprovechando así las ventajas del social media y creando una nueva oportunidad de negocio y de venta. Pero en este espacio no sólo se puede comprar los productos de Mark, también es un lugar para contactar entre compradoras y representantes de la marca así como para intercambiar consejos y experiencias muy valiosas para Mark.

Un caso un tanto contradictorio es el vivido entre BurgerKing y Facebook. Se trataba de una estrategia consistente en regalar una hamburguesa a aquellos usuarios que sacrificasen a diez de sus contactos (los eliminasen de su lista de amigos en Facebook). Aunque esta campaña solo tenía Estados Unidos como 
área de influencia, en su primera semana consiguió más de doscientas mil bajas, aunque finalmente $y$ debido a las presiones de Facebook, BurgerKing retiró la campaña ya que iba en contra de su filosofía eliminando canales de comunicación en lugar de crearlos.

Pero estos no son casos únicos, hay muchos más. Lufthansa, una de las mayores compañías de transporte aéreo del mundo posee una herramienta llamada MySkyStatus (myskystatus.com) que a través de Twitter y Facebook informa a tus contactos de la hora estimada de despegue y aterrizaje o de la altitud del avión, únicamente introduciendo previamente los datos del vuelo. Esto supone claramente una característica diferenciadora de la compañía frente a la competencia.

Adidas ha integrado Facebook y Google Maps (aplicación de google para el visionado de mapas) pare crear "el superlaser de la estrella de la Muerte de Star Wars", una aplicación con la que sobrevolar y atacar la ciudad, todo para promocionar una nueva colección inspirada en la película.

Starbucks, una de las cadenas de cafetería más grandes e importantes del mundo, promocionó durante el pasado mes de julio de 2009 su nueva línea de helados mediante una promoción que consistía en envíar cupones canjeables por tarrinas de helado. Durante toda la campaña promocional se repartieron casi 300.000 unidades. Pero esta no ha sido la única vez que Starbucks ha utilizado Facebook para comunicarse. El pasado 7 de diciembre de 2009 los usuarios de Facebook de 16 países (incluída España) fueron invitados a formar parte de una inciativa solidaria que consistía en cantar el famoso tema de The Beatles "All You Need Is Love" en lo que se conoce como Starbucks Love Project. Por cada vídeo subido a la red, la marca de café haría una donación a la ONG Red para luchar contra el SIDA en África. La relación entre Starbucks y Red ha generado unos resultados muy satisfactorios.

Save The Children Italia utilizó Facebook en la Navidad de 2009 con un notable éxito en una campaña destinada a conseguir donaciones destinadas a los niños de Africa. Algo parecido hicieron Famosa junto con ACB bajo en nombre "Encesta su juguete". Incluso el Museo Guggenheim de Bilbao ha organizado el concurso de fotografía "Frank Lloyd Wright: Concurso de fotografía de Arquitectura y Entorno", sobre arquitectura a nivel internacional utilizando la red social como soporte.

Otro ejemplo, es el de Panadol, uno de los productos analgésicos de la empresa farmaceutica GlaxoSmithKline (gsk). Conjuntamente con la agencia Memac OgilvyOne de Dubai elaboraron una estrategia de promoción en Facebook. Conscientes de que una de las herramientas más utilizadas en la red social es el etiquetado de amigos en fotos, desarrollaron una aplicación que bajo el nombre "Tag your moment" (etiqueta tu momento) permitía etiquetar las fotos con iconos, animaciones y música, creando así una mini historia animada.

Pero, algunas de estas acciones tienen los días contados, ya que según la Guía de Actuación de la red social, no se podrán promocionar en ella aquellos productos relacionados con el juego, el tabaco, los productos lácteos, las armas de fuego, los medicamentos o la gasolina, así como aquellos concursos cuyo premio entre dentro de las categorías citadas anteriormente. Medida que por supuesto ha provocado quejas entre los consumidores que se sienten incrédulos al ver como la red social compara las armas de fuego a los productos lácteos.

Esta Guía de Actuación añade que cualquier actividad de promoción que se lleve 
a cabo dentro de la red, deberá ser aprobada previamente y por escrito por los administradores de la red social.

\subsection{E nacimiento de un nuevo medio}

Llegado a este punto, podemos afirmar que las SRS no es un medio que utilizan algunos visionarios convencidos de su enorme potencial, sino todo lo contrario. Grupos de música, productoras de cine, bares y discotecas, marcas de gran consumo, tienen un pequeño espacio en Facebook. Los usuarios pueden hacerse fan de estos grupos, de las productoras, de las discotecas, de estas marcas... Acto seguido en su muro (lugar donde el usuario respondiendo a la pregunta "¿Qué estás pensando?" escribe acerca de su estado de ánimo, sobre lo que está haciendo o va a hacer...) aparece un mensaje que informa sobre esta afiliación, lo cual puede ser visto como un anuncio gratuito que todos los contactos del afiliado podrán ver. El marketing en Facebook consiste en comunicarse y no tanto en hacer publicidad, y a fin de cuentas, ino nos fiamos más de lo que nos diga un amigo que de lo que nos diga una marca?. Hoy en día, muchos anunciantes confían en Facebook como soporte complementario de sus campañas, consiguiendo así lo que conocemos como Marketing de $360^{\circ}$.

Después de este análisis, hemos podido constatar el potencial que tiene Facebook para las marcas y comprender el por qué de este boom.

Las redes sociales se presentan ante el futuro de las marcas como una herramienta casi imprescindible a la hora de realizar una investigación de mercados, test de productos, pretest y postest publicitarios. Esto significa, que se conoce la opinión del cliente solo con un clic, convirtiéndose en una herramienta de promoción sin fronteras.

El contenido de este concepto es algo tan sencillo que su magnitud casi se nos escapa de las manos. El conocimiento que tenemos sobre redes sociales, representa la punta de un iceberg de dimensiones desconocidas. Estas, se presentan ante las marcas como una de las herramientas de marketing más virales que pueden alcanzar a gran cantidad de clientes, tanto reales como potenciales y de forma muy rápida. Así, una vez que ambas partes están en contacto, las marcas podrán obtener todo tipo información que el usuario esté dispuesto a dar. Gustos, aficiones, costumbres, intereses, usos alternativos de productos, necesidades, tendencias presentes y futuras; en definitiva, todo aquello que una marca quiere saber, todo lo que una marca necesita para adaptarse a la demanda del mercado. Las redes sociales cuentan con la capacidad para llegar a cualquier lugar.

Todas las redes en general y Facebook en particular, se ponen al servicio de las marcas como un escaparate sin fín con el cuál darse a conocer ante el gran público y del cuál reciben información. Cualquier marca, puede crear un grupo en Facebook al que poco a poco se irán uniendo todos aquellos usuarios (clientes tanto reales como potenciales) que se sientan identificados con ella, consuman habitualmente sus productos o simplemente les guste. De esta forma, la marca dispone de una importantísima base de datos de clientes en la que, al menos aparentemente, se ha invertido el proceso tradicional, en lugar de llevar a cabo costosos y arduos procesos de segmentación de mercados en busca de un target, son ellos, los clientes los que poco a poco se van uniéndo.

Con la web 2.0, el mk 2.0 adquiere connotaciones de interactividad y personalización máximas a través de las cuáles, 
cada persona decide a qué grupos quiere pertenecer para recibir cierto tipo de información de acuerdo con sus intereses y necesidades, dejando atrás, las campañas publicitarias unidireccionales. Con las redes sociales, el mundo por fin ha comprendido que en esto de vender también interviene un individuo que tiene mucho que decir: tú.

Jugamos en un escenario de dimensiones desconocidas, pero no por ello, las marcas han de sentir vértigo dado que en este camino no están solas. Facebook ofrece un directorio de empresas y consultoras que pueden ayudarles, orientarles y ofrecerles toda clase de recursos para que entren con pie firme dentro de la red. Es lo que se llama el 'Preferred Developer Consultant Program', al cual pueden adherirse nuevas agencias y proveedores online que demuestren experiencia dentro de Facebook.

Según el Wall Street Journal y basándose en datos de NYPREX, la previsión de beneficios en 2010 para Facebook asciende a 710 millones de dólares, ¿de dónde sale todo este dinero?. Para profundizar un poco en este iceberg, podemos decir que Facebook no es una plataforma completamente gratuita. Las marcas al crear su página dentro de Facebook tienen dos posibilidades: una es la expuesta anteriormente y consiste en esperar para que poco a poco vaya aumentando el número de miembros y la otra, consiste en promocionar el sitio anunciándose. La primera vía utiliza un método similar al que explicabamos con la Teoría de los Seis Grados de Separación. Cuando un usuario se une a un grupo, sus contactos son informados de ello, si uno de ellos se une, los contactos de éste también lo verán y la repercusión será cada vez mayor. A través de la segunda vía, Facebook colocará un anuncio en función del perfil que previamente se haya fijado. Por ejemplo, con una segmentación mediante la cuál, se seleccionen a todos aquellos individuos ubicados en España, mayores de 18 años y con cualquier nivel educativo, la cifra de público a la que podíamos acceder a fecha diciembre de 2009 era de aproximadamente 7 millones de personas, con fecha febrero de 2010, la cifra supera los 7.800.000 personas (existen otros parámetros de segmentación como pueden ser el lugar de trabajo, situación sentimental, inclinaciones sexuales, idiomas...).

El coste de este servicio es el siguiente: se paga por los nuevos fans y por los clics. En primer lugar se establece un presupuesto diario, que como su propio nombre indica, es el máximo que la marca desea gastarse al día (el mínimo es $1 €$ ), en segundo lugar se establece una puja máxima (que supone el precio de cada clic), cada uno fija el precio que pagará por cada clic o por cada visualización del anuncio pero, claro está, cuanto mayor sea la puja mayores serán las oportunidades de que el anuncio aparezca. La puja mínima es de $0.01 €$, y para el segmento que creamos anteriormente, las pujas rondan entre los $0.17 €$ y los $0.24 €$ por $\mathrm{Clic}^{6}$.

¿Confían las marcas en Facebook? Parece que sí, o al menos confían en las redes sociales en su conjunto. Según un estudio de Forrester Research Inc., la inversión publicitaria en redes sociales fue de 716 millones de dólares en el año 2009 y es más, el estudio estima que ésta crecerá considerablemente hasta alcanzar en el año 2014 la cantidad de 3.113 millones de dólares con una tasa compuesta de crecimiento anual (CAGR) del 34\%, muy por delante de los servicios móviles que pasarán de 391 millones de dólares en 2009, a 1.274 millones de dólares en 2014 y un CAGR del $27 \%$.

6 La propia aplicación hace una estimación del valor de las pujas en función del target a través de lo que denomina "puja recomendada". 
Facebook expone tres casos reales de publicidad. El primero de ellos es de CM Photographic que se dirigía a mujeres entre 24 y 35 años cuya situación sentimental fuera "comprometidas". CM Photographic invirtió $600 \$$ y generó 40.000\$. Del total de usuarios que llegaron a la página de CM Photographics, el $60 \%$ se convirtieron en clientes potenciales y expresaron su interés por recibir más información. El segundo caso corresponde a StorQuest, empresa dedicada al alquiler de espacios de almacenaje. Con las estrategias de segmentación y apoyándose en las pujas en tiempo real consiguieron ampliar en un $50 \%$ el número de alquileres. El tercer y último caso real disponible en la propia web de Facebook es el de All Nippon Airlines. La aerolínea ideó un conjunto de palabras clave para dirigir su publicidad a usuarios que compartieran dos intereses, viajar y la cultura japonesa. Normalmente All Nippon Airlines conseguía entre un $8 \%$ y un $12 \%$ 208 de clics, en Facebook el resultado fue del $25 \%$.

Pero, ¿por qué pasa esto?. ¿Por qué reaccionamos ante un anuncio en Facebook de forma diferente ante el mismo anuncio por ejemplo en un periódico o en una revista?. La palabra clave en este caso es predisposición.

Cuando nos encontramos leyendo un periódico normalmente estamos relajados, sentados, sin excesivo ruido de fondo, prestamos atención a lo que estamos leyendo, a las imágenes... En este caso, la predisposición es mucho mayor que en el caso de la radio, por ejemplo, que muchas veces parece que nos acompaña como un sonido de fondo. Pero el caso de Facebook y de la publicidad en Internet en general, es diferente. Si mientras leemos las noticias, un anuncio en un periódico nos da la pista definitiva sobre eso que tenemos que comprar o ese regalo que tenemos que hacer, lo más que podemos hacer es pensar en ir a comprarlo, pero la posibilidad de dejar lo que estamos haciendo rara vez suele darse, en cambio, si nos encontramos conectados a una red social como puede ser Facebook y por casualidad vemos un anuncio en el que se nos ofrece un descuento especial en ese mismo regalo, la predisposición del propio medio nos llevará a hacer clic sobre él y puede que a adquirir el producto vía Internet. Ni que decir tiene que después del proceso de segmentación, las posibilidades de que finalmente se produzca ese clic son mayores.

¿Cómo hay que dirigirse hacia los usuarios de los SRS?. Antes de iniciar una campaña de comunicación destinada a las redes sociales debemos estudiar varios aspectos que hemos de tener siempre presentes. Al igual que en el mundo offline debemos tener claro qué es lo que queremos, de quién lo queremos y cómo pretendemos conseguirlo. Pero si algo marca la diferencia es el tono de comunicación empleado: si somos excesivamente formales, debido a las características del medio no conseguiremos llegar al target o público objetivo, mientras que si el tono es mucho más distendido la probabilidad de establecer fuertes canales de comunicación es mucho mayor. Son palabras claves del proceso de creación de la estrategia algunas como creatividad, diseño, personalización y sobre todo, interactividad. Una vez implantada es necesario un control y seguimiento que permita analizar los resultados y subsanar errores. Pero una cosa debemos de tener clara, no debemos buscar cantidad, sino calidad.

Recientemente ha salido al mercado La Biblia del Marketing de Facebook, la guía para comercializar con una marca, una compañía, un producto o un servicio en Facebook. Esta biblia tan particular está dividida en tres partes: herramientas de marketing de guerrilla, herramientas para anunciantes y herramientas para desarrolladores de aplicaciones, cada una de ellas explica los canales y vías disponibles para 
acceder al público objetivo o target a través de Facebook.

La primera parte sobre Marketing de Guerrilla habla sobre los perfiles, los grupos, las páginas, eventos, mensajes, noticias... La segúnda parte, sobre herramientas para anunciantes, expone los diferentes tipos de anuncios disponibles, la posibilidad de ofrecer regalos virtuales, estrategias de patrocinio... Por último, la tercera parte sobre las herramientas para desarrolladores de aplicaciones, se centra en estas aplicaciones: su diseño e implantación, estrategias de marketing para fomentar su uso, los sistemas de invitaciones y notificaciones, el desarrollo para tecnología móvil... En definitiva, una guía completa donde encontrar toda la información necesaria a la hora de hablar de Marketing en Facebook.

Finalmente una serie de datos $^{7}$ que las marcas han de tener presentes a la hora de llamar a la puerta de Facebook:

- Facebook aloja más de 350 millones de perfiles en todo el mundo, casi ocho millones y medio ubicados en España independientemente de su edad y referidos a enero de 2010. De todos ellos, un porcentaje bastante elevado entra al menos una vez al día.

- El mayor crecimiento se encuentra en usuarios de aproximadamente 35 años, el siguiente escalón es el de menores de 35.

- Cada usuario tiene aproximadamente 130 amigos.

- El mundo se pasa alrededor de seis billones de minutos al día conectados a Facebook. Google consigue la mitad.

- Los status se actualizan más de 40 millones de veces al día. Esto es, cada día 40 millones de personas quieren

\footnotetext{
7 Disponible en http://www.gabycastellanos.com/lo-que-nosabes-de-facebook-y-twitter/
}

contarle al mundo lo que pasa por su cabeza.

- Cada mes se suben millones de fotos y vídeos a Facebook.

- Las redes sociales están repartidas por todo el mundo. Tan solo el $30 \%$ de los usuarios se encuentran en Estados Unidos.

- Cada día más de 10 millones de usuarios se hacen fan de una página.

- Aproximadamente, 65 millones de usuarios acceden a Facebook a través de su móvil, los cuales son mucho más activos que los usuarios web.

\section{Resultados ¿visibles?}

Que las redes sociales pueden ayudar a la hora de llegar a nuestro target o público objetivo es evidente. Prueba de ello es que cada vez más redes sociales comienzan a ser rentables gracias al marketing, que ha visto en ellas un amplio escaparate del que muchos están pendientes. Pero ante todo, debemos tener en cuenta que la efectividad de las herramientas que utilicemos no depende únicamente de las características de éstas sino del uso que en cada caso se les dé, por lo que en ningún caso podremos achacar nuestros triunfos ni derrotas a nuestras herramientas sino a la forma de utilizarlas. Por lo tanto, una vez dicho esto, ¿debemos arriesgarnos al apostar por las redes sociales?, ¿efectivamente nos arriesgamos o vamos sobre seguro?

¿Es realmente fiable la repercusión que una marca puede conseguir? Nuevamente podemos hablar de casos reales. Durante las primeras semanas del año 2010, un fenómeno desconcertó a los hombres usuarios de Facebook que veían atónitos como algo estaba pasando. En principio parece que nadie sabía nada, lo único que pasaba es que todas las mujeres (o un alto porcentaje de ellas) respondían a la ya famosa pregunta "¿Qué estás pensando?" con un color. Evidentemente al darse 
cuenta de que no eran partícipes de algo que estaba revolucionando la red, se sentían interesados por el tema y preguntaban, me atrevo a decir que casi nunca hubo respuesta. Casi nunca hasta que hubo una filtración. Se trataba de un proceso viral destinado únicamente a mujeres que respondían al siguiente mensaje: "Escribe el color de tu sostén en el perfil. Sólo el color, nada más. Será interesante ver si ello extiende las alas de la conciencia sobre el cáncer de mama". A día de hoy (12 de enero de 2010) se desconoce cuál es el origen de este movimiento, aunque se tienen sospechas de que este se encuentre en una mujer bloguera de Detroit. ¿Cuántas mujeres han apoyado esta iniciativa? Es muy difícil calcularlo, por no decir imposible, pero uno de los grupos de apoyo en Facebook cuenta con 70.000 miembros.

Otro ejemplo muy similar, sobre la repercusión de los procesos de comunicación

en Facebook, es el caso del programa Click to Give (Cliquea para dar) de The Breast Cancer Site (www.thebreastcancersite.com), a través del cual se puede apoyar efectivamente la lucha contra el cáncer de mama haciendo clic sobre un botón rosa que directamente abre una página de anuncios (totalmente gratuito para el internauta). Con el dinero recaudado con este programa se hicieron en 2009 casi 7.000 mamografías gratuitas, que en términos reales suponen alrededor de 300.000 dólares.

Una prueba más de que las redes sociales se están afianzando no sólo en el resto del mundo, sino también en España, es el hecho de que Facebook acaba de inaugurar su oficina de ventas en nuestro país, desde la cuál se encargarán de integrar a las grandes marcas que operan en nuestro país en Facebook, pero además de estas grandes cuentas, también se mantendrá la dirigida a PYMES y la plataforma online que anteriormente hemos explicado.
Lo importante de todo esto, aparte de ejemplos que evidencian los resultados conseguidos, es la capacidad de respuesta de la sociedad. Es lógico que todo el mundo apoya la lucha contra el cáncer, además, si se pudiera comprobar que simplemente escribiendo un color se ayudase efectivamente a esta causa, el apoyo por parte de la sociedad probablemente sería masivo. ¿Sabes cuántas mujeres hay en Facebook?. Sólo en España hay más de 4.000 .000 de mujeres independientemente de su edad.

Nos referimos a los secretos a voces del social media porque las plataformas que las redes sociales ponen a disposición de los consumidores conforman una especie de confesionario donde exponer de forma relajada y sin aparente vigilancia todo aquello que queremos compartir. A no ser que hablemos de mensajes privados que evidentemente sólo puede ver la persona a la cual van dirigidos, el resto de comentarios son visibles cuanto menos para nuestros contactos.

Al introducir las marcas en este contexto pasa algo similar. Precisamente debido a la estructura del medio, a que nos encontramos relajados en nuestro hogar, sin presión (no como puede ocurrir, por ejemplo, en una dinámica de grupos o en una entrevista), todo ello hace que nuestro subconsciente esté predispuesto a hablar por nosotros y a expresar todo aquello que llevamos dentro con total libertad. Puede que esto resulte una escena un tanto bucólica e incluso algo exagerada, pero es precisamente este aspecto lo que marca la diferencia. Podemos decir que sólo en estos casos de relajación, si algo nos gusta lo decimos y si nos disgusta lo decimos aún más, y esa sensación de libertad nos impide pensar una y otra vez en si debemos opinar o no, o si esa opinión debemos expresarla de una u otra manera desaparece, consiguiendo en definitiva opiniones limpias y de un valor 
incalculable para nada sesgadas por "el qué dirán".

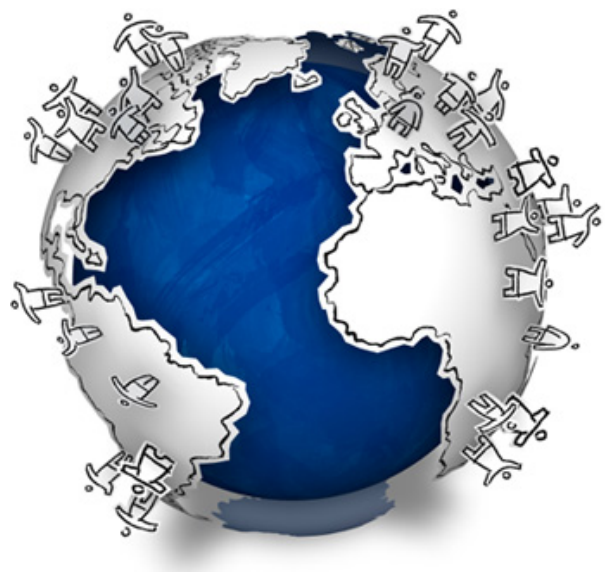

Sabemos a cuánta gente podemos llegar, y sabemos como hacerlo. Pero, irealmente conseguiremos llegar allí donde nos proponemos llegar?
Evidentemente, ni Facebook ni ninguna otra red social es una panacea publicitaria capaz de salvar todos los obstáculos que nos encontremos en el proceso de comercialización de nuestro producto, al igual que tampoco lo es ningún otro soporte publicitario, ¿o acaso podemos asegurar que si duplicamos nuestras ventas es debido a una nueva campaña en prensa o televisión?

Es cierto que muchos usuarios se sienten escépticos a la hora de hablar de la implantación de la publicidad dentro de los SRS, pero es imposible negar que la cantidad de tiempo que cada usuario pasa al día frente a ellas, no las transforme en una plataforma sumamente atractiva para los anunciantes.

\section{Bibliografía}

http://www.brandlife.es/2009/11/el-61-de-los-usuarios-de-redes-sociales-acceden-aellas-todos-los-dias/

http://blog.facebook.com/blog.php?post=190423927130

http://www.briefblog.com.mx/?p=9303

http://www.google.com/intl/en_us/press/zeitgeist2009/regional.html\#global

http://www.generacionesinteractivas.org/

http://www.20minutos.es/galeria/6437/0/0/mejores/fotos/dia/

http://www.rtve.es/noticias/20090212/los-usuarios-redes-sociales-permiten-quecualquiera-acceda-perfil/231173.shtml

http://www.maestrosdelweb.com/editorial/redessociales/

http://www.technorati.com/

http://apps.facebook.com/bloguzz/

http://www.brandlife.es/2009/12/pruebas-de-producto-en-facebook/

http://www.antena3noticias.com/PortalA3N/Ultraconectados-las-redes-

sociales/Facebook-suma-mas-300-millones-usuarios-gana-

dinero/EN 50146018345392

http://blogs.20minutos.es/quemepongo/post/2009/12/14/tavi-blogger-moda 
http://amis95.blogspot.com/

http://tavi-thenewgirlintown.blogspot.com/

http://blogs.20minutos.es/quemepongo/post/2009/12/14/tavi-blogger-moda

http://redessocialesblogs.suite101.net/article.cfm/top 10 aplicaciones de facebook http://www.tu-facebook.com/2009/02/cuantas-aplicaciones-hay-en-facebook.html http://manuelgross.bligoo.com/content/view/516215/El-uso-de-Facebook-como-

herramienta-de-marketing.html

http://www.facebook.com

http://www.insidefacebook.com/2008/08/10/the-facebook-marketing-bible-august-

2008-edition-is-now-available/

http://www.insidefacebook.com/la-biblia-del-mercadeo-en-facebook/

http://e-global.es/el-departamento-de-bomberos-de-los-angeles-utiliza-twitter-flickr-

youtube-blog-gabcast-jaiku.html

http://www.hoytecnologia.com/noticias/Obama-lidera-clubes-fans/94593

http://pagedata.insidefacebook.com/

http://www.elpais.com/articulo/tecnologia/Decenas/miles/mujeres/publican/color/so stenes/Facebook/elpeputec/20100111elpeputec 2/Tes

http://www.allfacebook.com/

http://lacolmenavirtual.wordpress.com/2010/01/09/las-mujeres-confiesan-el-color-

212 de-su-sujetador-en-facebook/

http://www.thebreastcancersite.com/

http://www.20minutos.es/noticia/593346/0/uso/redes/sociales/

http://es.wikipedia.org/wiki/Tuenti

http://sociedadinformacion.fundacion.telefonica.com/

http://www.tuenti.com

http://es.myspace.com

http://es.wikipedia.org/wiki/MySpace

http://www.brandlife.es/2010/01/avon-estrena-una-tienda-virtual-en-facebook-parasu-firma-mark/

http://www.briefblog.com.mx/2010/01/panadol-entra-a-facebook/

http://wiki.developers.facebook.com/index.php/Preferred_Developer_Consultant_Prog ram

http://www.brandlife.es/2009/12/facebook-ofrece-un-servicio-de-brand-consultants/

http://www.20minutos.es/noticia/602693/0/twitter/control/alcoholemia/

http://www.brandlife.es/2010/01/el-81-de-los-internautas-recuerda-la-publicidadgrafica-online-de-las-marcas/

http://www.e-global.es/medios-sociales-online/web-20-significa-internet-social-ycolaborativo.html

http://www.brandlife.es/2009/10/twitter-y-facebook-tambien-entre-nubes/ 
http://cent.uji.es/octeto/node/2115

http://www.forrester.com/Groundswell/profile_tool.html

http://www.thereagency.com/blog/tag/forrester/

http://www.onandoff.es/online social media marketing/crecimiento-de-inversionpublicitaria-en-redes-sociales/2009/12/11/

http://www.onandoff.es/online social media marketing/estrategia-en-redes-sociales-yii/2009/12/15/?utm source=feedburner\&utm medium=feed\&utm campaign=Fe ed\%3A+OnlineSocialMediaMarketing+(Online+Social+Media+Marketing)

http://www.onandoff.es/online social media marketing/estrategia-en-redes-socialesi/2009/12/14/?utm source=feedburner\&utm medium=feed\&utm campaign= Feed\%3A+OnlineSocialMediaMarketing+(Online+Social+Media+Marketing)

http://www.nexsa.cl/blog/?p=125

http://www.facebooknoticias.com/2010/01/05/campana-para-brindar-regalos-aninos-de-africa-es-un-exito/

http://www.facebooknoticias.com/2009/12/27/una-gran-campana-promovida-atraves-de-facebook/

http://www.facebooknoticias.com/2009/12/14/mark-zuckerberg-abre-perfil-publicoen-facebook/

http://www.underpc.com/articulos/36-internet/2219-facebook-no-anunciaremostabaco-armas-y-productos-lacteos

http://www.theinquirer.es/2009/11/29/no-es-facil-ser-popular-en-facebook.html

http://www.guggenheim-bilbao.es/microsites/frank lloyd wright/secciones/concur-

so fotografia/como participar.php?idioma=es

http://www.brandlife.es/2010/01/tiickr-nueva-plataforma-para-prescriptores-demarcas-via-redes-sociales/

http://www.elmundo.es/mundodinero/2010/01/26/economia/1264533312.html http://blog.addconsulta.pe/2009/10/camino-a-la-web-4-web-ubicua/

http://www.brandlife.es/2010/01/facebook-abre-oficina-de-ventas-en-espana/

http://www.redessociales10.com/los-conductores-ebrios-eluden-a-la-policia-con-twitter http://www.tecnologiablog.com/post/1525/facebook-google-maps-el-superlaser-de-laestrella-de-la-muerte-de-star-wars

http://www.twitter.com

http://www.tecnologiablog.com/post/1638/twitter-quiere-saber-donde-estas-trendingtopics-locales

http://www.cad.com.mx/historia_de_twitter.htm

http://www.tecnologiablog.com/post/1648/1648

http://www.puromarketing.com/53/6714/la-publicidad-twitter-le-va-encantar-todomundo-va-ser-alucinante.html

http://www.cad.com.mx/historia_de facebook.htm

http://www.gabycastellanos.com/espana-el-septimo-pais-en-uso-de-social-media/ 
Mª Aránzazu Sulé Alonso y Javier Prieto García

http://www.derechoeinternet.com/?s=red+social

http://www.fernandoplaza.com/2009/06/las-redes-sociales-son-los-centroscomerciales-de-intenet.asp

http://www.gabycastellanos.com/lo-que-no-sabes-de-facebook-y-twitter/

http://www.gabycastellanos.com/pepsi-se-olvida-de-la-publicidad-convencional-einvierte-20-millones-de-dolares-a-social-media/ 